\title{
Continuum extrapolation of finite temperature meson correlation functions in quenched lattice QCD
}

\author{
Anthony Francis* \\ Fakultät für Physik, Universität Bielefeld, D-33615 Bielefeld, Germany \\ E-mail: afrancisephysik.uni-bielefeld.de

\section{Frithjof Karsch} \\ Physics Department, Brookhaven National Laboratory, Upton, NY 11973, USA \\ E-mail: karschdbn1.gov
}

\begin{abstract}
We explore the continuum limit $a \rightarrow 0$ of meson correlation functions at finite temperature. In detail we analyze finite volume and lattice cut-off effects in view of possible consequences for continuum physics. We perform calculations on quenched gauge configurations using the clover improved Wilson fermion action. We present and discuss simulations on isotropic $N_{\sigma}^{3} \times 16$ lattices with $N_{\sigma}=32,48,64,128$ and $128^{3} \times N_{\tau}$ lattices with $N_{\tau}=16,24,32,48$ corresponding to lattice spacings in the range of $0.01 \mathrm{fm} \lesssim a \lesssim 0.031 \mathrm{fm}$ at $T \simeq 1.45 T_{c}$. Continuum limit extrapolations of vector meson and pseudo scalar correlators are performed and their large distance expansion in terms of thermal moments is introduced. We discuss consequences of this analysis for the calculation of the electrical conductivity of the QGP at this temperature.
\end{abstract}

The XXVIII International Symposium on Lattice Field Theory, Lattice2010

June 14-19, 2010

Villasimius, Italy

\footnotetext{
* Poster contribution.

† Speaker.
} 


\section{Introduction}

The heavy-ion experiments at RHIC and soon LHC are probing deeper and deeper into the high temperature region of QCD. As such more and more experimental results are presented that require a more complete knowledge of finite temperature QCD. Naturally one turns to lattice QCD to give some answers to the questions arising. However some questions of interest are notoriously difficult to answer from a lattice perspective. This is in particular the case for the analysis of spectral properties of hadron correlation functions. Although here the main interest is in extracting information on hadronic spectral functions at low frequencies, this cannot be achieved without good control over the large frequency region. Correlation functions thus need to be controlled at short as well as large distances. High accuracy data on large lattices at several values of the cutoff are needed to control finite volume and lattice cut-off effects. Only then a reliable continuum extrapolation becomes possible which is a prerequisite for extracting dependable physics results.

In this combined talk and poster contribution we present results from a lattice analysis of meson correlation functions at finite temperature [四]. We emphasize here our systematic analysis of the finite volume and cut-off dependence of thermal meson correlation functions. This analysis leads to the conclusion that we indeed can take the continuum limit for the vector and pseudo scalar current correlation functions in a large Euclidean time interval. We also calculate several thermal moments of hadron spectral functions to better explore the spectral properties at low frequencies. We close with a discussion of the constraints arising from our analysis for the determination of the electrical conductivity in the quark gluon plasma at finite temperature [■], 目].

\section{Meson Correlation Functions}

Our key observable of interest is the Euclidean correlation function of a given particle current,

$$
J_{v} \equiv \bar{q}(\tau, \vec{x}) \gamma_{v} q(\tau, \vec{x})
$$

where choosing the appropriate gamma matrix we obtain the vector particle channels for $\gamma_{v}=\gamma_{\mu}$ where $\mu=0, \ldots, 4$ and the pseudo scalar for $\gamma_{v}=\gamma_{5}$.

In this work we analyze the Euclidean temporal two-point correlation function for the above channels at fixed momentum,

$$
G_{\mu v}(\tau, \vec{p})=\sum_{\vec{x}} G_{\mu v}(\tau, \vec{x}) e^{i \vec{p} \cdot \vec{x}}
$$

where

$$
G_{\mu v}(\tau, \vec{x})=\left\langle J_{\mu}(\tau, \vec{x}) J_{v}^{\dagger}(0, \overrightarrow{0})\right\rangle
$$

while we denote thermal expectation values with $\langle\ldots\rangle$. In this work we will set the momentum to zero, $\vec{p}=0$, and suppress the second argument in the hadronic correlation functions.

At high temperatures the spectrum of meson resonances is more and more changed by thermal effects; the width of resonances will broaden and the onset of the continuum in the spectrum may 
shift. As a result the interest in the analysis of the above current-current correlation function shifts to their representation in terms of a spectral function $\rho_{\mu v}$ [四]:

$$
G_{\mu v}(\tau, T)=\int_{0}^{\infty} \frac{d \omega}{2 \pi} \rho_{\mu v}(\omega, T) \frac{\cosh (\omega(\tau-1 / 2 T))}{\sinh (\omega / 2 T)} .
$$

From here on we denote with $\mu v \equiv i i$ the sum over the space-like components of the vector spectral function $\rho_{i i}$, while the full vector spectral function is given by $\rho_{V} \equiv \rho_{00}+\rho_{i i}$. We also use the notation $P S$ for $\mu \nu \equiv 55$. Note that the correlation function $G_{00}(\tau, T)$ is connected to the net number of quarks $(q-\bar{q})$ in a given flavor channel, i.e., $n_{0}(\tau)=\int d^{3} x J_{0}(\tau, \vec{x})$. As the net quark number is conserved, it does not depend on time, $n_{0}(\tau)=n_{0}$. Thus the corresponding correlation function is constant in Euclidean time and the spectral function is simply given by,

$$
\rho_{00}(\omega)=-2 \pi \chi_{q} \omega \delta(\omega),
$$

with the quark number susceptibility

$$
\chi_{q}=-\int d^{3} x\left\langle J_{0}(\tau, \vec{x}) J_{0}^{\dagger}(0, \overrightarrow{0})\right\rangle .
$$

Consequently the time-like correlation function obeys the relation $G_{00}(\tau, T) \equiv-\chi_{q} T$, which immediately leads to an exact relation between $G_{i i}(\tau, T)$ and $G_{V}(\tau, T)$ :

$$
G_{i i}(\tau, T)=\chi_{q} T+G_{V}(\tau, T) .
$$

In the free field limit the spectral functions are seen to increase quadratically for large frequencies $\omega$. One obtains:

$$
\begin{aligned}
\rho_{i i}(\omega) & =2 \pi \chi_{q} \omega \delta(\omega)+\frac{3}{2 \pi} \omega^{2} \tanh (\omega / 4 T) \\
\rho_{V}(\omega) & =\rho_{00}(\omega)+\rho_{i i}(\omega)=\frac{3}{2 \pi} \omega^{2} \tanh (\omega / 4 T) \\
\rho_{P S}(\omega) & =\frac{3}{4 \pi} \omega^{2} \tanh (\omega / 4 T)
\end{aligned}
$$

At finite temperature the exact cancellation of the $\delta$-functions in the $\rho_{V}(\omega)$ spectral function is only approximate, as $\rho_{00}(\omega)$ continues to be proportional to a $\delta$-function due to the connection with net quark number, while the $\delta$-function in $\rho_{i i}(\omega)$ is subject to thermal effects. As a consequence this contribution is smeared out into a Breit-Wigner shaped peak [వ]:

$$
\rho_{i i}(\omega) \rightarrow \rho_{i i}^{B W}(\omega)=2 \chi_{q} c_{B W} \frac{\omega \Gamma / 2}{\omega^{2}+(\Gamma / 2)^{2}}+(1+\kappa) \cdot \frac{3}{2 \pi} \omega^{2} \tanh (\omega / 4 T),
$$

where $\kappa$ parametrizes corrections to the high frequency behavior of the free field limit. This smearing in the low frequency region is directly related to the appearance of finite transport coefficients in the thermal medium, e.g. the electrical conductivity,

$$
\frac{\sigma}{T}=\frac{C_{e m}}{6} \lim _{\omega \rightarrow 0} \frac{\rho_{i i}(\omega)}{\omega T},
$$

where $C_{e m}$ is the sum over the squared charges of the contributing quark flavors. The pseudo scalar spectral function on the other hand does not per se contain an additional low frequency contribution; a connection with transport phenomena is not expected. 


\section{Thermal Moments of the Correlation Function}

A useful set of observables that helps to characterize the structure of spectral functions, is given by "thermal moments" of the spectral function at vanishing momentum. At a given order we define these moments as the Taylor expansion coefficients of the correlation function expanded around the midpoint of the Euclidean time interval ${ }^{1}$, i.e., around $\tau T=1 / 2$,

$$
G_{H}^{(2 n)}=\left.\frac{1}{(2 n) !} \frac{d G_{H}(\tau T)}{d(\tau T)^{2 n}}\right|_{\tau T=1 / 2}=\frac{1}{(2 n) !} \int_{0}^{\infty} \frac{d \omega}{2 \pi}\left(\frac{\omega}{T}\right)^{2 n} \frac{\rho_{H}(\omega)}{\sinh (\omega / 2 T)}
$$

and

$$
G_{H}(\tau T)=\sum_{n=0}^{\infty} G_{H}^{(2 n)}\left(\frac{1}{2}-\tau T\right)^{2 n} .
$$

The infinite temperature, free field limit of the correlation function can be calculated analytically. For massless quarks one obtains [6]:

$$
\begin{aligned}
G_{V}^{\text {free }}(\tau T)=2 G_{P S}^{\text {free }}(\tau T) & =6 T^{3}\left(\pi(1-2 \tau T) \frac{1+\cos ^{2}(2 \pi \tau T)}{\sin ^{3}(2 \pi \tau T)}+2 \frac{\cos (2 \pi \tau T)}{\sin ^{2}(2 \pi \tau T)}\right) \\
G_{i i}^{\text {free }}(\tau T) & =T^{3}+G_{V}^{\text {free }}(\tau T)
\end{aligned}
$$

Using these results the first three non-vanishing moments are then given by

$$
\begin{aligned}
& G_{V}^{(0), \text { free }}=2 G_{P S}^{(0), \text { free }}=\frac{2}{3} G_{i i}^{(0), \text { free }}=2 T^{3}, \\
& G_{H}^{(2), \text { free }}=2 G_{P S}^{(2), \text { free }}=\frac{28 \pi^{2}}{5} T^{3}, \\
& G_{H}^{(4), \text { free }}=2 G_{P S}^{(4), \text { free }}=\frac{124 \pi^{4}}{21} T^{3} .
\end{aligned}
$$

In our lattice simulation we will also analyze ratios of moments. In the free field limit they are given by

$$
\begin{aligned}
R_{V, \text { free }}^{(2,0)} & =\frac{G_{V}^{(2), \text { free }}}{G_{V}^{(0), \text { free }}}=R_{P S, \text { free }}^{(2,0)}=\frac{14 \pi^{2}}{5} \simeq 27.635 \\
R_{\text {ii, free }}^{(2,0)} & =\frac{2}{3} R_{V, \text { free }}^{(2,0)} \simeq 18.423 \\
R_{H, \text { free }}^{(4,2)} & =\frac{155 \pi^{2}}{147} \simeq 10.407,
\end{aligned}
$$

which can be obtained from the ratio of the mid-point subtracted correlation functions and the corresponding free field values:

$$
\frac{\Delta_{H}(\tau T)}{\Delta_{H}^{\text {free }}(\tau T)}=\frac{G_{H}(\tau T)-G_{H}^{(0)}}{G_{H}^{\text {free }}(\tau T)-G_{H}^{(0), \text { free }}}=\frac{G_{H}^{(2)}}{G_{H}^{(2), \text { free }}}\left(1+\left(R_{H}^{(4,2)}-R_{H, \text { free }}^{(4,2)}\right)\left(\frac{1}{2}-\tau T\right)^{2}+\ldots\right) \cdot(3.1
$$

Note that the distinction between $H=i i$ or $H=V$ is unnecessary when evaluating $\Delta_{H}(\tau T)$, as differences in hte correlators, which arise from contributions of $\delta$-functions in the spectral functions, are eliminated in subtracted correlators.

\footnotetext{
${ }^{1}$ As the spectral functions as well as the integration kernel in the spectral representation of the correlators are odd functions of the frequency, all odd moments vanish.
} 


\begin{tabular}{|c|c|c|c|c|c|c|c|c|c|c|}
\hline$N_{\tau}$ & $N_{\sigma}$ & $\# \operatorname{conf}$ & $\beta$ & $a[\mathrm{fm}]$ & $T / T_{c}$ & $c_{S W}$ & $\kappa$ & $m_{\overline{M S}} / T_{[\mu=2 \mathrm{Gev}]}$ & $Z_{V}$ & $Z_{P S}$ \\
\hline 48 & 128 & 451 & 7.793 & 0.010 & 1.43 & 1.3104 & 0.13340 & $0.1117(2)$ & 0.861 & 0.79 \\
\hline 32 & 128 & 255 & 7.457 & 0.015 & 1.45 & 1.3389 & 0.13390 & $0.0989(4)$ & 0.851 & 0.78 \\
\hline 24 & 128 & 340 & 7.192 & 0.021 & 1.42 & 1.3673 & 0.13431 & $0.1062(2)$ & 0.842 & 0.76 \\
& & 156 & & & & & 0.13440 & $0.02367(5)$ & & \\
\hline 16 & 128 & 191 & 6.872 & 0.031 & 1.46 & 1.4125 & 0.13495 & $0.02429(5)$ & 0.829 & 0.74 \\
& 64 & 191 & & & & & & & & \\
& 48 & 229 & & & & & & & & \\
& 32 & 251 & & & & & & & & \\
\hline
\end{tabular}

Table 1: Simulation parameters for the generation of gauge field configurations on lattices of size $N_{\sigma}^{3} \times N_{\tau}$.

\section{Simulation Parameters and Results}

\subsection{Computational Details}

Our numerical results are obtained from quenched QCD gauge field configurations generated with the standard SU(3) single plaquette Wilson gauge action [ [ $]$ ]. Using an over-relaxed heat bath algorithm configurations were generated on lattices of size $N_{\sigma}^{3} \times N_{\tau}$, where $32<N_{\sigma}<128$ and $N_{\tau}=16,24,32$ and 48 , with a separation of 500 updates per configuration. Correlation functions and plaquette expectation values calculated on these configurations have been checked and are seen to be uncorrelated. Details on the statistics collected are given in Tab. I.

All calculations presented here have been performed at a single temperature value, $T \simeq 1.45 T_{c}$. The gauge couplings have been adjusted accordingly for the different temporal lattice sizes. To do so we extrapolate known results for the critical coupling $\beta_{c}\left(N_{\tau}\right)$ and the square root of the string tension $\sqrt{\sigma}[\mathbb{8}]$. This is achieved by fitting $T / \sqrt{\sigma}$ using the ansatz suggested in [Q] within the range of $\beta \in[5.6,6.5]$ and extrapolating to our relevant region of $\beta \in[6.8,7.8]$. All simulation parameters are given in Tab. W.

In the fermion sector we employ the clover improved Wilson action with non-perturbatively chosen clover coefficient $c_{S W}[\mathbb{W}]$ and determine the correlation functions using an even-odd decomposed CG-algorithm. The hopping parameter $\kappa$ is chosen to be close to its critical value [U]] and tuned to give approximately constant quark masses for four of the examined lattices. Here the quark masses are estimated using the axial Ward identity (AWI) to calculate the AWI current quark mass $m_{A W I}$ for the different values of cut-off. Whereby we use the non-perturbatively improved axial vector current with coefficient $c_{A}$ noted in [12]. In the next step we calculate the renormalization group invariant mass $m_{R G I}$ and rescale to the commonly quoted $\overline{M S}$-scheme at the scale $\mu=2 \mathrm{GeV}$. The results are also listed in Tab. .

\subsection{Numerical Results}

Due to the subtlety of the effects in question it is not very illuminating to examine the correlator directly. To illustrate this we show the correlation functions of the vector and pseudo scalar currents together with their analytically obtained free continuum and free discretized, lattice counterparts in Fig. $\mathbf{~}$. Clearly the exponential decay of the correlation function dominates and obscures all 

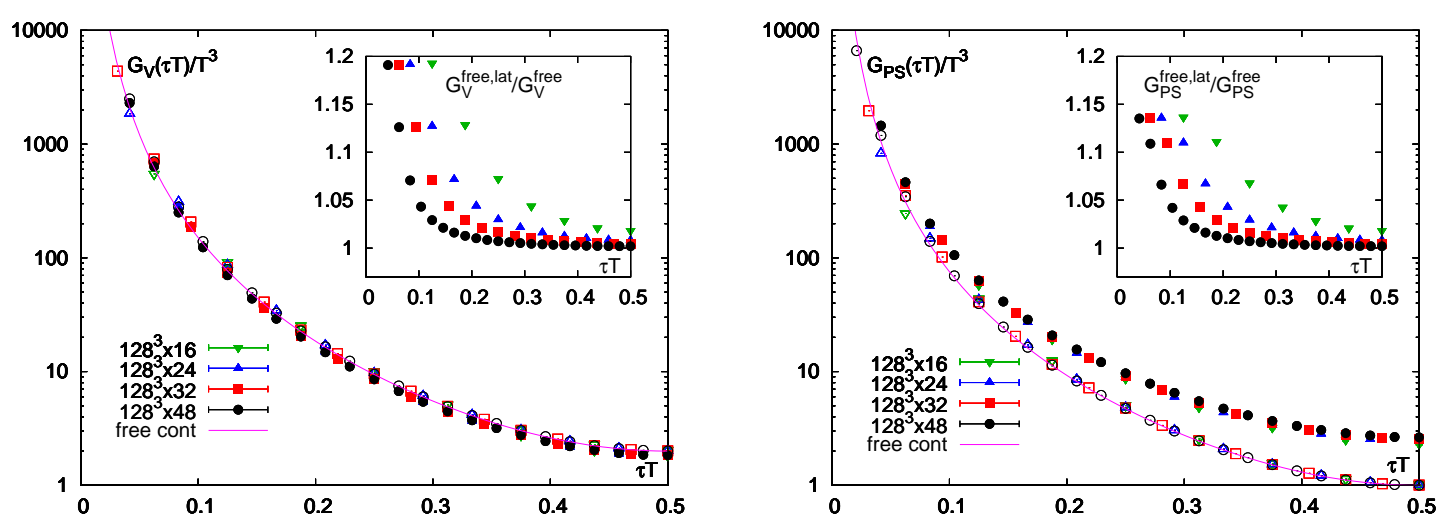

Figure 1: The vector (left) and pseudo scalar (right) correlation functions together with their free continuum (magenta lines) and free lattice (open symbols) counterparts. (Blow-ups): The ratio of free lattice and free continuum correlation functions.

other interesting physics features. To circumvent this effect it is useful to look at certain ratios that largely cancel the exponential part of the Euclidean time dependence of the correlators.

One of these ratios is that of the correlation function itself divided by the free correlation function given in Eq. [.3.3,

$$
R_{H}(\tau, T)=\frac{G_{H}(\tau, T)}{G_{H}^{f r e e}(\tau, T)}
$$

The immediate advantage of a ratio as that given in Eq. 4. 1 is that it gives a direct handle on the deviation of the correlation function calculated at finite and infinite temperature, respectively. As the continuum as well as lattice free correlation functions are known analytically, this also provides insight into the importance of lattice cut-off effects as function of Euclidean time. The insertions

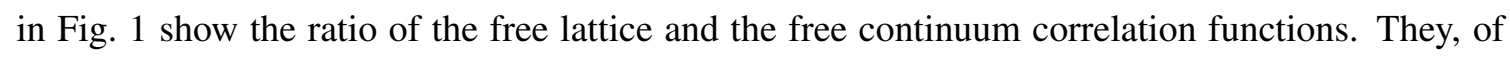
course, show that cut-off effects are largest at small distances. Moreover, it becomes obvious that with increasing cut-off values (larger $N_{\tau}$ ) the onset of cut-off effects is shifted to smaller distances.

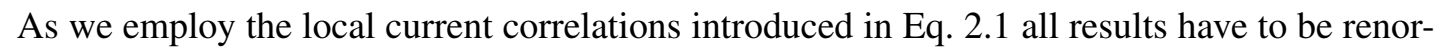
malized multiplicatively,

$$
J_{v}^{l a t}=\left(2 \kappa Z_{H}\right) \bar{q}(\tau, \vec{x}) \gamma_{v} q(\tau, \vec{x})
$$

where the renormalization constants have been determined non-perturbatively for the vector [ए]] and perturbatively up to two-loop order for the pseudo scalar channel [ए3]]. They are listed in Tab. 四. In the case of the vector correlation functions the above statement holds true also for the time-like component $G_{00}(\tau, T)$ because the local lattice current is not conserved at non-zero lattice spacing. Consequently the time-like correlation function $G_{00}(\tau, T)$ may not be strictly $\tau$-independent. However, as all vector current-current correlation functions are subject to the same renormalization constants a rescaling of the correlation functions $G_{V}(\tau, T)$ and $G_{i i}(\tau, T)$ or even the ratio $R_{H}(\tau, T)$ by $G_{00}(\tau, T)$ yields a quantity independent of renormalization. 


\begin{tabular}{|c|c|c|c|c|c|}
\hline$N_{\tau}$ & 16 & 24 & 32 & 48 & $\infty$ \\
\hline$\chi_{q} / T^{2}$ & $0.882(10)$ & $0.895(16)$ & $0.890(14)$ & $0.895(8)$ & $0.897(3)$ \\
\hline
\end{tabular}

Table 2: Quark number susceptibility $\left(\chi_{q} / T^{2}\right)$ calculated on lattices of size $128^{3} \times N_{\tau}$. The quark number susceptibilities have been renormalized using the renormalization constants listed in Tab. 1. The last column gives the result of a continuum extrapolation taking into account cut-off errors of $\mathscr{O}\left(a^{2}\right)$.
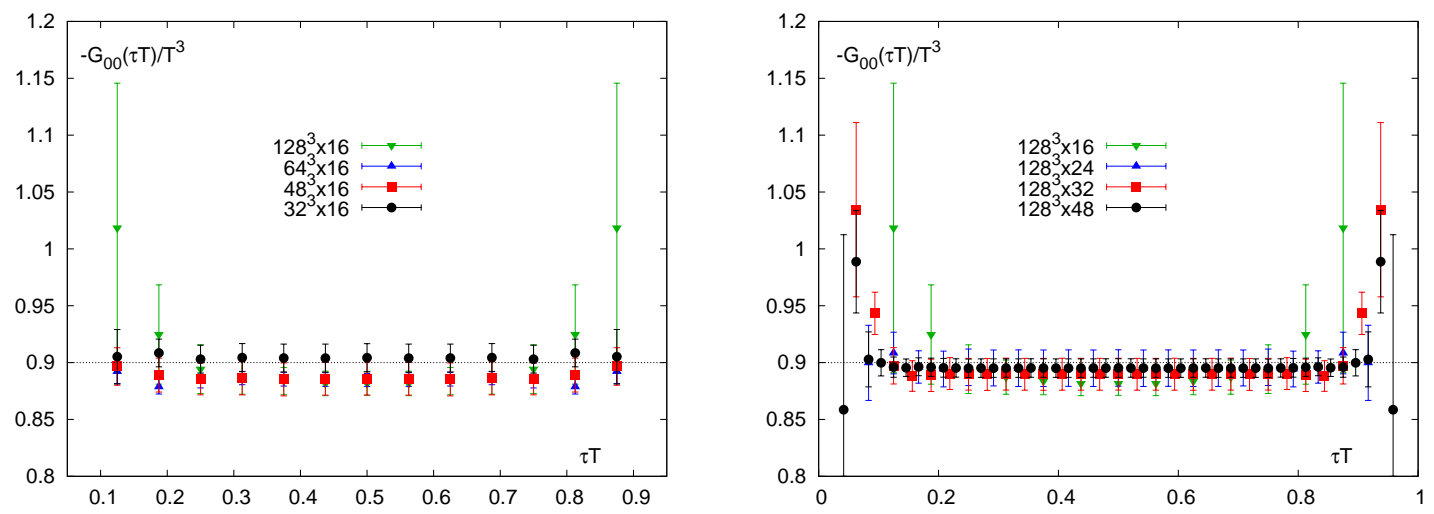

Figure 2: The time-like component of the vector spectral function, $G_{00}(\tau T) / T^{3}$, calculated at $T \simeq 1.45 T_{c}$. The left hand part of the figure shows the volume dependence of $G_{00}(\tau T) / T^{3}$ for $N_{\tau}=16$ and $32 \leq N_{\sigma} \leq$ 128. The right hand figure shows the cut-off dependence of $G_{00}(\tau T) / T^{3}$ for $N_{\sigma}=128$ and $16 \leq N_{\tau} \leq 48$.

\subsection{Finite Volume and Lattice Effects}

\subsubsection{Quark Number Susceptibility}

As mentioned above the time-like component of the vector correlation function, $G_{00}(\tau T)$, will be used for rescaling in the following, so it is the first channel to be tested for finite volume and cut-off effects. We find these effects to be small, as can be seen from Fig. 凤, where we plot $-G_{00}(\tau T) / T^{3}$. The quark number susceptibility may then be extracted from the long distance behavior of the correlator, $\chi_{q} / T^{2}=-G_{00}(\tau T \simeq 0.5) / T^{3}$.

On the left of Fig. $\square$ we show the results for the $N_{\tau}=16$ lattice with fixed cut-off while varying the spatial extent. Except for aspect ratio $N_{\sigma} / N_{\tau}=2$ all results agree within statistical errors at the level of $1 \%$. On the right the cut-off dependence at fixed spatial extent $N_{\sigma}=128$ is shown. Here too systematic effects in the quark number susceptibility are seen to be small.

Note even though we implement the non-conserved local current of the time-like vector correlation function, we obtain results that are $\tau$-independent to a very high degree. The deviations at small distances $\tau T$ might be understood as lattice effects. The results for the quark number susceptibility are summarized in Tab. \.

\subsubsection{Vector and Pseudo Scalar Correlation Functions}

Turning to the vector and pseudo scalar correlation functions we stress that the large range of available spatial lattice sizes at fixed cut-off, with aspect ratios ranging between $2 \leq N_{\sigma} / N_{\tau} \leq 8$, allows to quantify finite volume effects. Moreover, the large temporal extent of maximum $N_{\tau}=48$ 

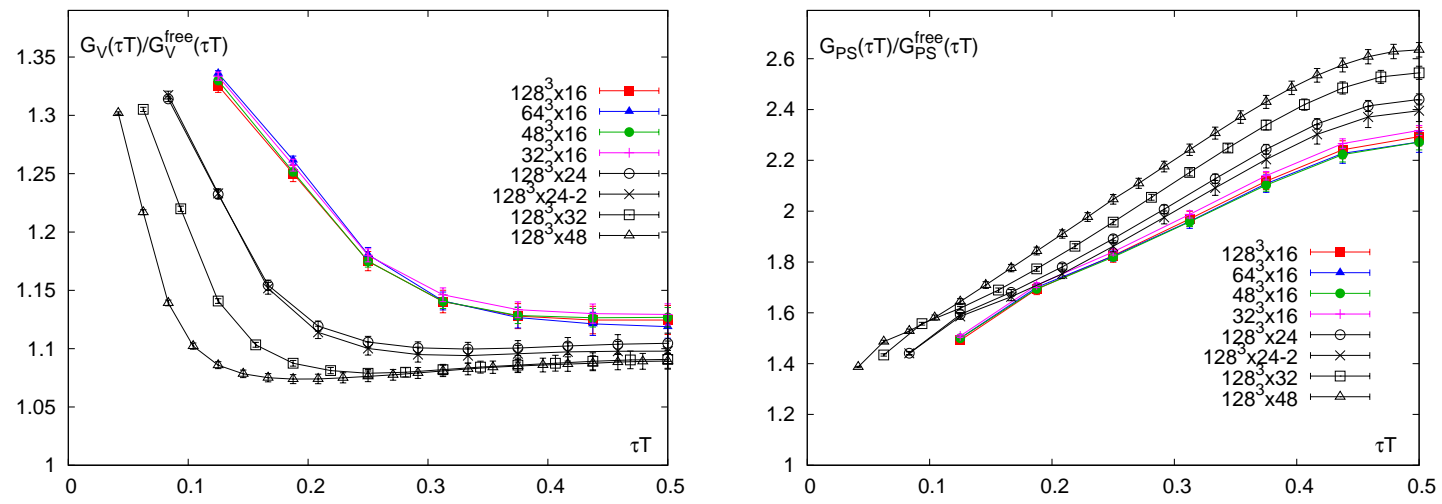

Figure 3: The vector (left) and pseudo scalar (right) correlation functions, calculated on lattices of size

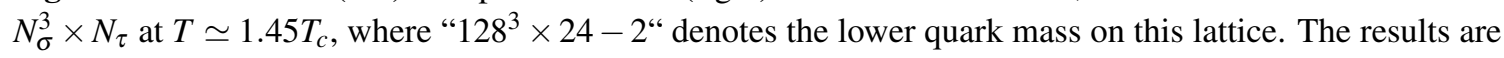
normalized using the corresponding free correlation functions. Shown are data for $\tau T>1 / N_{\tau}$ only.

on an isotropic (!) lattice reduces the lattice spacing to $0.01 \mathrm{fm}$ at $T \simeq 1.45 T_{c}$. The variation of $N_{\tau}$ by up to a factor of three allows to control lattice cut-off effects. In the following it will be shown that finite volume effects in the correlation functions are well under control and a controlled extrapolation to the continuum limit is indeed possible in a large Euclidean time interval. Note that that our current analysis improves on systematic errors that were present in earlier calculations of the vector spectral function performed by employing the same discretization scheme [144].

In Fig. [3 we show results for the vector and pseudo scalar correlation functions using the ratios introduced in Eq. 4 . On the left hand side the ratio for $H=V$ and on the right hand side for $H=P S$ is shown for all available lattice sizes. Data sets with fixed spatial size at $N_{\sigma}=128$ while varying the cut-off $N_{\tau}$ shown in black. Data sets with fixed cut-off $\left(N_{\tau}=16\right)$ and varying volume are shown in color. For one value of the cut-off $\left(N_{\tau}=24\right)$ we performed calculations for two different values of the quark masses. We find that finite quark mass effects are small and well within $2 \%$. From the fixed cut-off (colored) $N_{\tau}=16$ results in both plots finite volume effects for $\tau T \geq 0.3$ are seen to remain within a few percent even for the largest Euclidean time separation at $\tau T=0.5$. As a consequence these results show that finite volume effects are under control. For $\tau T<0.3$ in the left $H=V$ plot it is immediately apparent that cut-off effects are large in the ratio.

In the pseudo scalar case the situation concerning cut-off effects is not immediately evident. The ratio shown in Fig. B(right) shows large deviations from the free field behavior even at short distances. At all distances the correlator thus seems to be controlled by large non-perturbative effects. Moreover, the analysis of cut-off effects is obscured by the fact that data have been rescaled by using renormalization constants, which are known only perturbatively. This introduces unknown uncertainties. To eliminate at least these uncertainties we show in Fig. [t the pseudo scalar correlation function normalized by the pseudo scalar correlation function at $\tau T=0.5$. As we focus on the cut-off dependence we only show equal quark mass $N_{\sigma}=128$ results. The left hand figure shows the pseudo scalar correlator normalized by free continuum correlator and in the right hand figure the free lattice correlation function has been used. From the left hand plot the cut-off dependence becomes immediately evident and we conclude that, similarly to the vector case, cut-off effects above $\tau T=0.3$ are small and increase with decreasing $\tau T$. Even though their effect becomes ap- 

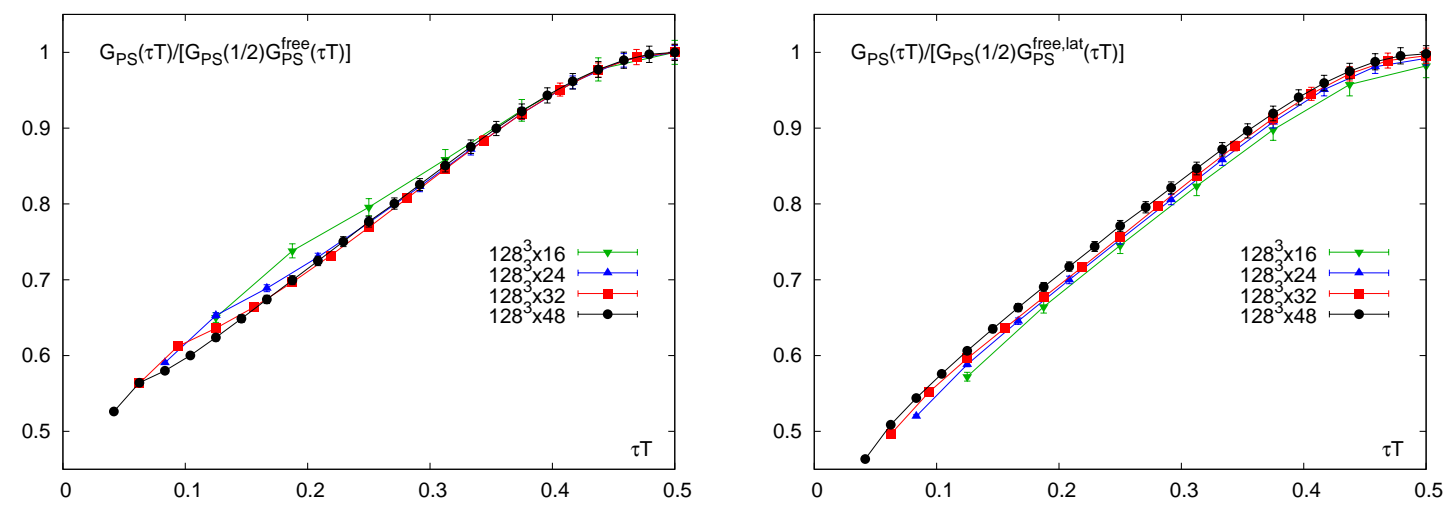

Figure 4: The pseudo scalar correlation function normalized by the free continuum (left) and free lattice (right) correlation functions, both additionally rescaled by the correlator at $\tau T=1 / 2, G_{P S}(\tau T=0.5)$.

parent for $\tau T \leq 0.3$, they do not dominate the behavior of the correlation function as in the vector channel. Actually the right hand side of Fig. 团indicates that the $\tau$-dependence of the cut-off effects is similar to that of the free lattice correlation functions, as any cut-off effect is hardly visible also for $\tau T \leq 0.3$.

\subsection{Continuum Extrapolation}

The results shown in the previous section indicate that the continuum limit can be taken for the vector correlation functions at least for $\tau T \gtrsim 0.25$. To do so we use a quadratic ansatz in $a T=1 / N_{\tau}$ to fit ratios of the vector correlator and the corresponding free field values. We normalize these ratios using the quark number susceptibility $\chi_{q} / T^{2}$ and perform fits at fixed temporal extent $\tau T$. As cut-off effects are large on the $N_{\tau}=16$ lattice we will only use data from the $N_{\tau}=24,32,48$ lattices, for reference we will however include the former in our figures.

In Fig. [] we show results of this extrapolation in $1 / N_{\tau}^{2}$ for $\tau T=1 / 2,7 / 16,3 / 8,5 / 16$ and $1 / 4$ where we used the free continuum as well as the free lattice correlation functions for normalization. Note that we performed extrapolations for all Euclidean times $\tau T$ available on the $N_{\tau}=48$ lattice. Wherever the smaller lattices fail to have a corresponding point in $\tau T$ we interpolate using a spline construction. Subsequently the errors are then calculated using a Jackknife-method. In Fig. [1 this is the case for $\tau T=7 / 16$ and 5/16. The figure reveals that the continuum limit can be cleanly taken and consistent results are obtained by using the free continuum and the free lattice normalizations, respectively.

In the left hand part of Fig. 6 we show the results of the extrapolation in the vector channel as described above. Note that the largest deviation from the free correlation function occurs at $\tau T=1 / 2$. In fact, the established bending of the vector correlation function is crucial for a quantitative description of the low frequency region of the vector spectral function in terms of the ansatz suggested in Eq. 2.Tl. The short distance part of the correlation function obviously can be well described by the free spectral function including the correction factor $(1+\kappa)$ as also has been done in Eq. 2.].

In the right hand part of Fig. 6 the corresponding result for the pseudo scalar correlation function is shown. Here it is not possible to suppress the renormalization effects using suitable ratios 


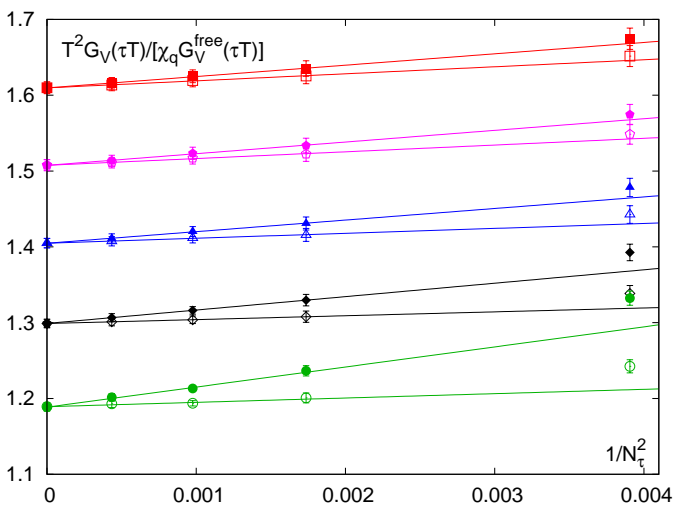

Figure 5: The ratio $R_{V}(\tau T)$ normalized by the quark number susceptibility using the free continuum (full symbols) and lattice (open symbols) correlation functions over $1 / N_{\tau}^{2}$. Shown are the results of $\tau T=1 / 2,7 / 16,3 / 8,5 / 16$ and $1 / 4$, for legibility the results have been offset by 0.1 respectively. For $\tau T=7 / 16$ and 5/16 spline interpolations were used to estimate the corresponding results on the $N_{\tau}=24$ lattice.
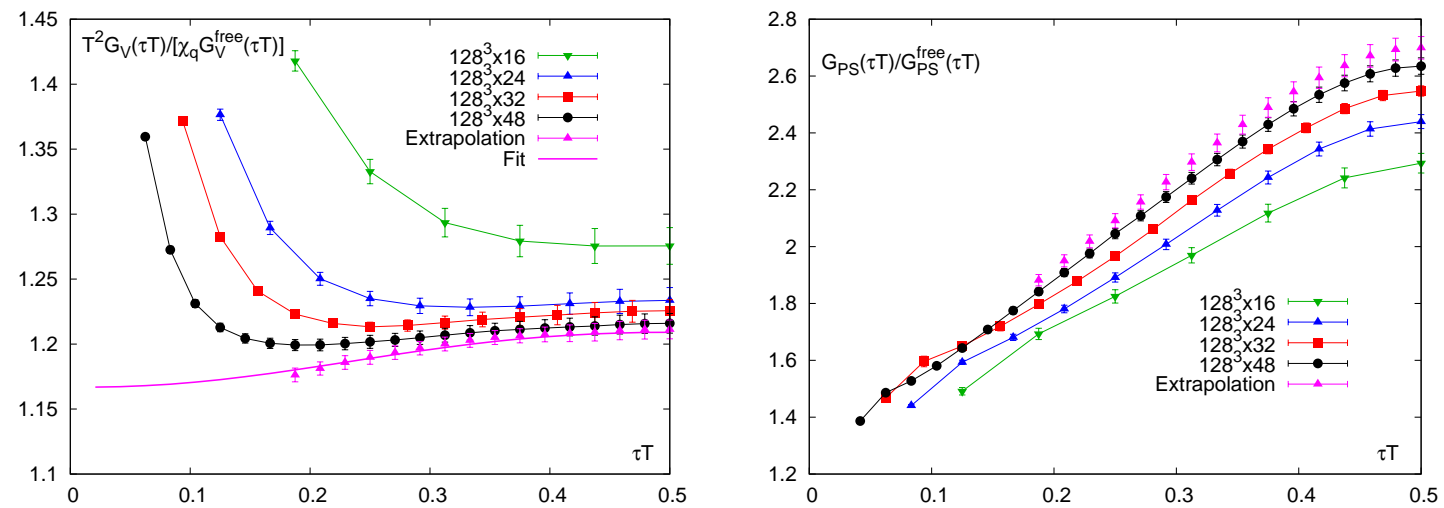

Figure 6: (left): The ratio $R_{V}(\tau T)$ normalized by the quark number susceptibility using the free continuum correlation functions over $\tau T$ and its corresponding continuum extrapolation. (right): The unnormalized $R_{P S}(\tau T)$ including its continuum extrapolation. In both cases the extrapolation was done as described, filling in spline interpolations when necessary. The solid curve in the left hand figure shows the fit discussed in section 5 .

of correlation functions. The extrapolation necessarily also includes this ambiguity. As the correlator normalized by its value at the midpoint was found to be almost cut-off independent and as finite volume effects were seen to be small renormalization effects dominate the uncertainty of the extrapolation.

\subsection{Curvature of the Vector and Pseudo Scalar Correlation Functions}

As discussed in section 3, thermal moments give additional insight into the spectral representation of hadronic correlation functions. They are especially interesting as they are obtained at the largest Euclidean time separation where the correlation functions are most sensitive to the low frequency region of the spectral function. In particular, the lower orders of the thermal moments 
restrict the magnitude of the low frequency contribution to the spectral function and thus to the correlation function.

In order to extract thermal moments we examine the quantity $\Delta_{H}(\tau T)$ defined in Eq. B.]. Once more we rescale this ratio of subtracted correlators by the quark number susceptibility. In Fig. $\square$ we show results obtained from the vector and pseudo scalar correlators, respectively. We perform an extrapolation of $\Delta_{H}(\tau T)$ to the continuum, exactly as outlined for the correlation functions themselves. The extrapolated data is then fitted to a quartic polynomial as indicated in the Taylor-expansion in Eq. 3 .] to obtain $\Delta_{H}(\tau T)$ at $\tau T=1 / 2$. In the vector channel this gives

$$
\frac{T^{2} G_{H}^{(2)}}{\chi_{q} G_{H}^{(2), \text { free }}}=1.1916 \pm 0.0011
$$

where $H$ may denote either $i i$ or $V$ as noted in section 3. Combining these results with those of the continuum extrapolation for the vector correlation functions we obtain the ratios $R_{V}^{(2,0)}$ and $R_{i i}^{(2,0)}$,

$$
R_{V}^{(2,0)}=27.187 \pm 0.286<R_{V, \text { free }}^{(2,0)} \text { and } R_{i i}^{(2,0)}=19.217 \pm 0.193>R_{i i, f r e e}^{(2,0)} .
$$

Repeating this analysis also in the pseudo scalar channel we obtain $\Delta_{P S}(\tau T) / G_{P S}^{(0)}$ shown in the right hand part of Fig. $\square$ and the following results for the second moment,

$$
\frac{T^{3} G_{P S}^{(2)}}{G_{P S}^{(0)} G_{P S}^{(2), \text { free }}}=0.7912 \pm 0.0012, \text { thus } \quad R_{P S}^{(2,0)}=10.932 \pm 0.017<R_{P S, \text { free }}^{(2,0)} .
$$

These results reveal some interesting properties of the individual thermal moments; a combination of Eqs. 4.3 and 4.4 indicates that the second thermal moment is closer to the free field value for the $H=V$ case and farther away for the $H=i i$, respectively. To evaluate Eq. 4.5 in this way we need $G_{P S}^{(0)} / G_{P S}^{(0), \text { free }}$, which from Fig. 6 can be seen to be larger than 1 . Subsequently, even though we are not able to extract the latter quantity without fully controlling renormalization effects, we can conclude the second moment must be closer to the free field limit than the zeroth.

In both channels we also tried to examine the fourth thermal moment, but our numerical results unfortunately do not permit a conclusive determination of this value.

\section{Electrical Conductivity}

The results obtained for the vector correlation function and its continuum extrapolation, as well as the result on the second thermal moment put stringent bounds on the magnitude and shape of any contribution to the low frequency behavior of the vector spectral function. The small deviations from the free vector correlation function also suggest that the spectral function of the free theory is a good starting point for an analysis of the vector spectral function at finite temperature. We thus used as an ansatz for the spectral function the form given in Eq. 2.J]. This ansatz depends on four temperature dependent parameters: the quark number susceptibility $\chi_{q}(T)$, which we already extracted from the time-like component of the vector correlation function, the strength $\left(c_{B W}(T)\right)$ and width $(\Gamma(T))$ of the Breit-Wigner contribution and the higher order corrections to the high frequency free field spectral function, which we parametrize at present by a constant $\kappa(T)$. Already 

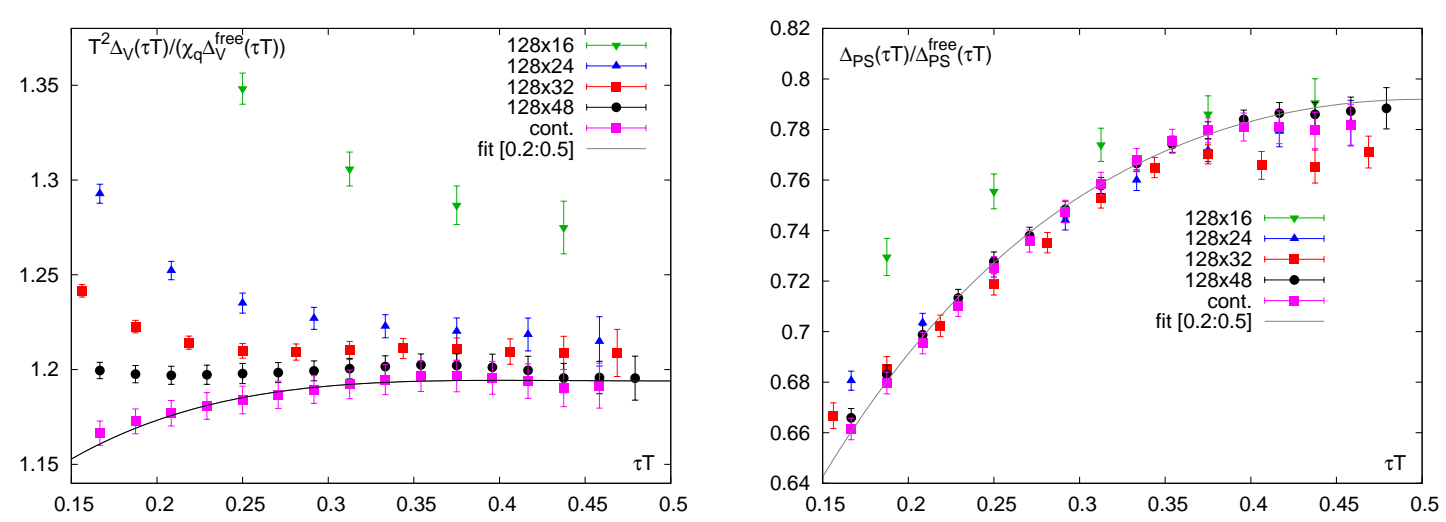

Figure 7: (left): The mid-point subtracted vector correlation function normalized to the corresponding difference for the free vector correlation function. Shown is $\Delta_{V}(\tau T)$ but normalized by the quark number susceptibility. (right): The pseudo scalar case $\Delta_{P S}(\tau T)$ normalized by $G_{P S}^{(0)}$. The Fits in both Figures obey a quartic ansatz as indicated by the definition of $\Delta_{H}(\tau T)$ and are shown within the interval $\tau T \in[0.2: 0.5]$.

with this ansatz we obtain good fits for both the spatial $\left(G_{i i}\right)$ and vector $\left(G_{V}\right)$ correlation functions. In fact, a combined fit to the continuum extrapolated vector correlation function in the Euclidean time interval $[0.25: 0.5]$ and the second thermal moment, gives excellent results with a $\chi^{2} / d o f$ below unity. For details on the fitting procedure and a more elaborate discussion of the results we refer to [四]. The parameters obtained using this ansatz are:

$$
2 c_{B W} T / \Gamma=1.235 \pm 0.056, \Gamma / T=2.23 \pm 0.15,(1+\kappa)=1.046 \pm 0.004 .
$$

This fit is shown in Fig. $\mathrm{G}(\mathrm{left})$. Of course, as a consequence of this fit ansatz we also obtain a result for the behavior of the spectral function close to $\omega=0$, i.e. we can deduce the electrical conductivity of the quark gluon plasma at $T \simeq 1.45 T_{c}$ :

$$
\frac{\sigma}{T}=\frac{C_{e m}}{6} \lim _{\omega \rightarrow 0} \frac{\rho_{i i}(\omega)}{\omega T}=\frac{2 C_{e m}}{3} \cdot \frac{c_{B W} \chi_{q}}{\Gamma}=(0.37 \pm 0.02) \cdot C_{e m} .
$$

We stress, however, that this result is a consequence of the particular ansatz used to fit the vector correlation function. An important question is, of course, to what extent this ansatz is unique or allows for modifications, in particular at low energies, which will influence the determination of the electrical conductivity. We intend to address this question by performing fits within a larger class of spectral functions as well as the Maximum Entropy Method [四].

\section{Summary}

We have presented a detailed analysis of light meson correlation functions at $T \simeq 1.45 T_{c}$ in quenched QCD. For the vector current channels we find that finite volume and cut-off effects are under good control in a large Euclidean time interval. Here it is possible to take the continuum limit. The calculation of the second thermal moment and its inclusion in fits greatly helped to constrain the fit parameters. This led to an estimate of the electrical conductivity of the QGP at $T \simeq 1.45 T_{c}$, the significance of which requires further investigations in a larger parameter space. 
In the pseudo scalar channel deviations from free field behavior are much more pronounced. In particular the analysis of cut-off effects is more difficult, as the perturbatively computed renormalization constants introduce additional systematic uncertainties. However, rescaling the results by the pseudo scalar correlation function at the midpoint yields a largely cut-off independent result. Also finite size effects are found to be small in the pseudo scalar channel. This suggests that a spectral analysis of the pseudo scalar correlation functions should yield reliable results for its frequency dependence and may suffer only somewhat from an imprecise knowledge of the overall normalization. We will address the spectral analysis of the pseudo scalar correlator elsewhere.

\section{Acknowledgments}

This work has been supported in part by contract DE-AC02-98CH10886 with the U.S. Department of Energy and by grant GRK 881 of the Deutsche Forschungsgemeinschaft. Numerical simulations have been performed on the BlueGene/P at the New York Center for Computational Sciences (NYCCS) which is supported by the U.S. Department of Energy and by the State of New York and the BlueGene/P at the John von Neumann Supercomputer center (NIC) at FZ-Jülich,Germany.

This presentation is to a large extent based of joint work with Heng-Tong Ding, Olaf Kaczmarek, Edwin Laermann and Wolfgang Soeldner. We thank them for all their important input to this work.

\section{References}

[1] H.-T. Ding, A. Francis, O. Kaczmarek, F. Karsch, E. Laermann and W. Soeldner, to be published.

[2] S. Gupta, Phys. Lett. B 597, 57 (2004).

[3] G. Aarts, C. Allton, J. Foley, S. Hands and S. Kim, Phys. Rev. Lett. 99, 022002 (2007).

[4] F. Karsch and H. W. Wyld, Phys. Rev. D 35, 2518 (1987).

[5] G. Aarts and J. M. Martinez Resco, Nucl. Phys. B 726, 93 (2005).

[6] W. Florkowski and B.L. Friman, Z. Phys. A347 (1994) 271.

[7] K.G. Wilson, Phys. Rev. D 10 (1974), 2445.

[8] B. Lucini, M. Teper and U. Wenger, JHEP 0401, 061 (2004).

[9] C. R. Allton, Lattice Monte Carlo data versus perturbation theory, arXiv:heplat/ 9610016.

[10] M. Lüscher, S. Sint, R. Sommer, P. Weisz and U. Wolff, Nucl. Phys. B491 (1997) 323.

[11] M. Lüscher, S. Sint, R. Sommer and H. Wittig, Nucl. Phys. B491 (1997) 344.

[12] M. Guagnelli and R. Sommer, Nucl. Phys. Proc. Suppl. 63, 886 (1998).

[13] A. Skouroupathis and H. Panagopoulous, Phys. Rev. D 78, 119901(E) (2008); A. Skouroupathis and H. Panagopoulous, Phys. Rev. D 79, 094508 (2009)

[14] F. Karsch, E. Laermann, P. Petreczky, S. Stickan and I. Wetzorke, Phys. Lett. B 530, 147 (2002). 